\title{
REPRESENTATIONS OF FROBENIUS-TYPE TRIANGULAR MATRIX ALGEBRAS
}

\author{
FANG LI CHANG YE
}

\begin{abstract}
The aim of this paper is mainly to build a new representation-theoretic realization of finite root systems through the so-called Frobenius-type triangular matrix algebras by the method of reflection functors over any field. Finally, we give an analog of APRtilting module for this class of algebras. The major conclusions contains the known results as special cases, e.g. that for path algebras over an algebraically closed field [7] and for path algebras with relations from symmetrizable cartan matrices [11]. Meanwhile, it means the corresponding results for some other important classes of algebras, that is, the path algebras of quivers over Frobenius algebras and the generalized path algebras endowed by Frobenius algebras at vertices.
\end{abstract}

Key words: Frobenius-type triangular matrix algebras, reflection functor, locally free module, root system, APR-tilting module

\section{Introduction and Preliminaries}

Let $Q$ be a finite connected acyclic quiver, and let $H=k Q$ be the path algebra of $Q$ for an algebraically closed field $k$. Gabriel showed that the quiver $Q$ is representation-finite if and only if $Q$ is a Dynkin quiver of type $A_{n}, D_{n}, E_{6}, E_{7}, E_{8}$ in [8]. In this case, there is a bijection between the isomorphism classes of indecomposable representations of $Q$ and the set of positive roots of the corresponding simple complex Lie algebra. Bernstein, Gelfand and Ponomarev introduced the machinery of Coxeter functors, which are defined as compositions of reflection functors, to give an elegant proof of Gabriel's theorem in [5]. Gabriel also showed that there are functorial isomorphisms $S C^{ \pm} \cong \tau^{ \pm}(-)$in [9], where $S$ is a twist functor and $\tau(-)$ is the Auslander-Reiten translation. Auslander, Platzeck and Reiten showed that there exists an $H$-module $T$ satisfying the functorial isomorphisms $F_{k}^{ \pm}(-) \cong H_{o m}(T,-)$ in [4] for a BGP-reflection functor $F_{k}^{ \pm}$and the APR-tilting module $T$.

Some of these results have been developed to valued graphs or $k$-species by Dlab and Ringel for a field $k$, see [18], [6], [7]. Moreover, in [11], for any field $k$, Geiss, Leclerc and Schröer generalized them to a class of 1-Gorenstein algebras $A$, which were defined via quivers with relations associated to symmetrizable Cartan matrices, as follows.

Mathematics Subject Classification(2010): 16G10, 16G20, 16G60

Date: version of July 16, 2021. 
Let $C=\left(c_{i j}\right) \in M_{n}(\mathbb{Z})$ be a symmetrizable generalized Cartan matrix with a symmetrizer $D=\operatorname{diag}\left(c_{1}, \cdots, c_{n}\right)$. For all $c_{i j}<0$, write that $g_{i j}:=\left|\operatorname{gcd}\left(c_{i j}, c_{j i}\right)\right|, f_{i j}:=\left|c_{i j}\right| / g_{i j}, k_{i j}:=$ $\operatorname{gcd}\left(c_{i}, c_{j}\right)$.

An orientation of $C$ is a subset $\Omega \subset\{1,2, \ldots, n\} \times\{1,2, \ldots, n\}$ such that the following hold:

(i) $\{(i, j),(j, i)\} \cap \Omega \neq \emptyset$ if and only if $c_{i j}<0$;

(ii) For each sequence $\left(\left(i_{1}, i_{2}\right),\left(i_{2}, i_{3}\right), \ldots,\left(i_{t}, i_{t+1}\right)\right)$ with $t \geq 1$ and $\left(i_{s}, i_{s+1}\right) \in \Omega$ for all $1 \leq s \leq t$ we have $i_{1} \neq i_{t+1}$.

For an orientation $\Omega$ of $C$, let $Q:=Q(C, \Omega):=\left(Q_{0}, Q_{1}, s, t\right)$ be the quiver with the set of vertices $Q_{0}:=1, \ldots, n$ and the set of arrows

$$
Q_{1}:=\left\{\alpha_{i j}^{(g)}: j \rightarrow i \mid(i, j) \in \Omega, 1 \leq g \leq g_{i j}\right\} \cup\left\{\varepsilon_{i}: i \rightarrow i \mid 1 \leq i \leq n\right\} .
$$

For a qiuver $Q=Q(C, \Omega)$ and a symmetrizer $D=\operatorname{diag}\left(c_{1}, \ldots, c_{n}\right)$ of $C$, let

$$
A=A(C, D, \Omega):=k Q / I
$$

where $k Q$ is the path algebra of $Q$, and $I$ is the ideal of $k Q$ defined by the following relations:

(i) For each $i$ we have the nilpotency relation $\varepsilon_{i}^{c_{i}}=0$.

(ii) For each $(i, j) \in \Omega$ and each $1 \leq g \leq g_{i j}$ we have the commutativity relation $\varepsilon_{i}^{f_{j i}} \alpha_{i j}^{(g)}=$ $\alpha_{i j}^{(g)} \varepsilon_{j}^{f_{i j}}$.

It was proved in [11] that the algebra $A$, given in (1) is of 1-Gorenstein.

The aim of this paper is to give a more larger class of 1-Gorenstein algebras in which the important conclusions about representation theory still hold.

In the sequel, the ground field $k$ is always permitted to be any field.

For $n \geq 2$, define a triangular matrix algebra $\Gamma$ of order $n$ satisfying $\Gamma=\left(\begin{array}{cccc}A_{1} & A_{12} & \ldots & A_{1 n} \\ 0 & A_{2} & \ldots & A_{2 n} \\ \vdots & \vdots & \ddots & \vdots \\ 0 & 0 & \ldots & A_{n}\end{array}\right)$, where each $A_{i}$ is an algebra and $A_{i j}$ an $A_{i}$ - $A_{j}$-bimodule with bimodule maps $\mu_{i l j}: A_{i l} \otimes_{A_{l}}$ $A_{l j} \rightarrow A_{i j}$ such that the following diagram commutes:

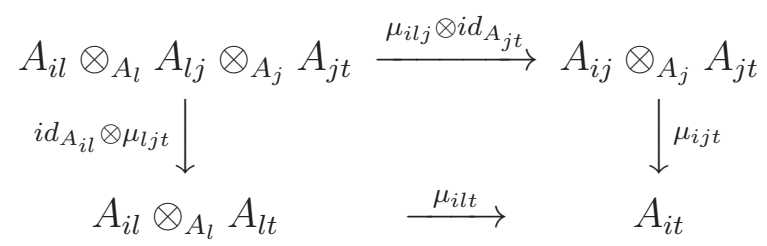

for $1 \leq i<l<j<t \leq n$, whose multiplication is given by $(A B)_{i j}=\sum_{i<l<j} \mu_{i l j}\left(a_{i l} \otimes b_{l j}\right)$ for $A=\left(a_{i j}\right)_{n \times n}, B=\left(b_{i j}\right)_{n \times n} \in \Gamma$ where $(A B)_{i j}$ means the $(i, j)$-entry of $A B$. 
A representation $X$ of $\Gamma$ is defined as a datum $X=\left(\begin{array}{c}X_{1} \\ X_{2} \\ \vdots \\ X_{n}\end{array}\right)_{\phi_{i j}}$, with $\phi_{i j}: A_{i j} \otimes_{A_{j}} X_{j} \rightarrow X_{i}$ an $A_{i}$-module morphism for $1 \leq i<j \leq n$ so that it satisfies the following commutative diagram:

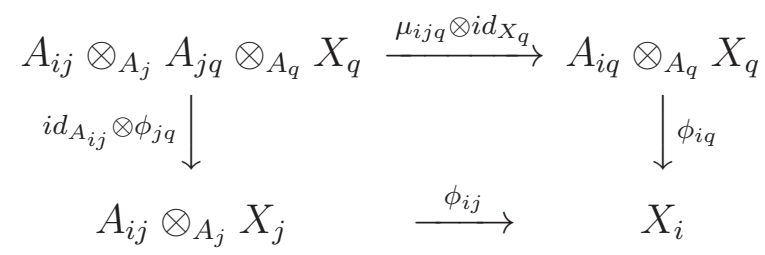

A morphism $f$ from a representation $X$ to another representation $Y$ is defined as a datum $\left(f_{i}, 1 \leq i \leq n\right)$, where $f_{i}: X_{i} \rightarrow Y_{i}$ is an $A_{i}$-map such that $f_{i} \phi_{i j}=\phi_{i j}\left(i d_{A_{i, j}} \otimes f_{j}\right)$ for each $1 \leq i<j \leq n$.

Then we obtain the representation category of $\Gamma$, denoted as $\operatorname{Rep}(\Gamma)$.

Definition 1.1. Let $A_{i}$ be Frobenius algebras with units $e_{i}$ for all $i=1, \cdots, n$. Let $B_{i j}$ be $A_{i}$ $A_{j}$-bimodules as free left $A_{i}$-modules and free right $A_{j}$-modules of finite ranks respectively

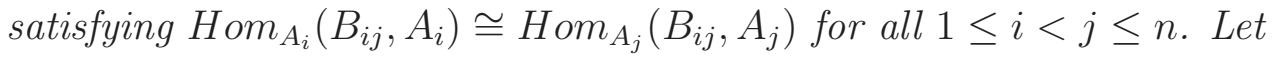

$$
A_{i j}=\oplus_{l=0}^{j-i-1} \oplus_{i<k_{1}<k_{2}<\cdots<k_{l}<j} B_{i k_{1}} \otimes_{A_{k_{1}}} B_{k_{1} k_{2}} \otimes \cdots \otimes_{A_{k_{l}}} B_{k_{l} j}
$$

for $1 \leq i<j \leq n$, where $l=0$ means the direct summand $B_{i j}$.

Define a triangular matrix algebra $\Lambda=\left(\begin{array}{cccc}A_{1} & A_{12} & \ldots & A_{1 n} \\ 0 & A_{2} & \ldots & A_{2 n} \\ \vdots & \vdots & \ddots & \vdots \\ 0 & 0 & \ldots & A_{n}\end{array}\right)$ which is called a Frobeniustype triangular matrix algebra, if it satisfies (2) where the map $\mu_{i j q}: A_{i j} \otimes_{A_{j}} A_{j q} \rightarrow A_{i q}$ is the natural inclusion map.

The algebra $A=A(C, D, \Omega)=k Q / I$ in (1) from [11] is indeed a Frobenius-type triangular matrix algebra. To see this, one needs only to re-order vertexes by $i<j$ if $(i, j) \in \Omega$ and take $A_{i}=e_{i} H e_{i}, B_{i j}=A_{i} \operatorname{Span}_{k}\left(\alpha_{i j}^{(g)} \mid 1 \leq g \leq g_{i j}\right) A_{j}$. It is easy to see such $A$ satisfy the condition of Frobenius-type triangular matrix algebra in Definition 1.1.

Following this fact, in this paper the major results on Frobenius-type triangular matrix algebras are the improvement of the corresponding ones in [11]. For convenience, in the sequel, we will always assume $A_{i}$ to be finite dimensional for all $i=1, \cdots, n$. 
Remark 1.2. (i) In Definition 1.1, if each $A_{i}$ is only a finite dimension algebra, and the condition $\operatorname{Hom}_{A_{i}}\left(B_{i j}, A_{i}\right) \cong \operatorname{Hom}_{A_{j}}\left(B_{i j}, A_{j}\right)$ is replaced by that $H_{o m}\left(B_{i j}, A_{i}\right)$ is a projective $A_{j}$-module or an injective $A_{j}$-module, then $\Lambda$ is called a normally upper triangular gm algebra, which was introduced and investigated in [16].

Since $\operatorname{Hom}_{A_{j}}\left(B_{i j}, A_{j}\right)$ is a free left $A_{j}$-module and $\operatorname{Hom}_{A_{i}}\left(B_{i j}, A_{i}\right) \cong \operatorname{Hom}_{A_{j}}\left(B_{i j}, A_{j}\right)$ we obtain that $\operatorname{Hom}_{A_{i}}\left(B_{i j}, A_{i}\right)$ is a free left $A_{j}$-module, then, of course, a projective $A_{j}$-module. So all Frobenius-type triangular matrix algebras are normally upper triangular gm algebras.

(ii) In Definition 1.1, let $B_{i j}=0$ for all $|i-j| \geq 2$ and but $A_{i}$ are allowed to be any rings, then we obtain a class of triangular matrix rings which were studied in [20] about their module category and some homological characterizations.

(iii) A path algebra of quiver over algebra $\Lambda=A Q=A \otimes_{k} k Q$ for an acyclic quiver $Q$ and a Frobenius algebra $A$ which studied in [19] is also a Frobenius-type triangular matrix algebra. Here one needs only to take all $A_{i}=A$ and $B_{i j}=\oplus_{s=1}^{\#\{\alpha: j \rightarrow i\}} A$. In particular, it was investigated in [19] for the case $A=k[T] /\left[T^{2}\right]$ the algebra of dual numbers.

(iv) A generalized path algebra $\Lambda=k(Q, \mathcal{A})$, with an acyclic quiver $Q, \mathcal{A}=\left\{A_{i}\right\}_{i \in Q_{0}}$ and Forbenius algebras $A_{i}\left(i \in Q_{0}\right)$, is a Frobenius-type triangular matrix algebra through taking all $B_{i j}$ to be generalized arrows from $j$ to $i$ and thus obtaining $A_{i j}$ as generalized paths from $j$ to $i$. For more details, see [16].

Let $X=\left(\begin{array}{c}X_{1} \\ X_{2} \\ \vdots \\ X_{n}\end{array}\right)_{\phi_{i j}}$ be a $\Lambda$-module, it is easy to see that $\phi_{i j}: A_{i j} \otimes_{A_{j}} X_{j} \rightarrow X_{i}$ is uniquely determined by $\left.\phi_{i j}\right|_{\operatorname{Res}\left(B_{i j} \otimes X_{j}\right)}: B_{i j} \otimes_{A_{j}} X_{j} \rightarrow X_{i}$ for $1 \leq i<j \leq n$.

Remark 1.3. Without ambiguity, we sometimes omit to write $\phi_{i j}$, especially when $\phi_{i j}$ is a natural inclusion for all $1 \leq i<j \leq n$.

Remark 1.4. In this paper, we consider only $\Lambda$ which is connected, that is, $\Lambda$ can NOT be written as $\left(\begin{array}{cc}\Lambda_{1} & 0 \\ 0 & \Lambda_{2}\end{array}\right)$ for two non-zero Frobenius-type triangular matrix algebras $\Lambda_{1}$ and $\Lambda_{2}$. Otherwise, then $\Lambda=\left(\begin{array}{cc}\Lambda_{1} & 0 \\ 0 & \Lambda_{2}\end{array}\right)$, whose any representation $M$ is always a direct sum representation $M_{i}$ of $\Lambda_{i}(i=1,2)$, that is, $M=M_{1} \oplus M_{2}$.

This article is organized as follows. In Section 2, we study some properties of Frobeniustype triangular matrix algebras and in particular, show that they are a class of 1-Gorenstein algebras. In Section 3, we define the reflection functors for Frobenius-type triangular matrix algebras and give the relation between Coxeter functors and Auslander-Reiten translation for 
these algebras. In Section 4, we recall some definitions and basic facts on Cartan matrices, quadratic forms and Weyl groups. Then we give a new representation-theoretic realizations of all finite root systems via Frobenius-type triangular matrix algebras. In Section 5, we study the generalized versions of $A P R$-tilting modules over Frobenius-type triangular matrix algebras.

\section{LoCAlly Free MOdUles AND 1-Gorenstein PROPERTY}

Proposition 2.1. [16] The representation category of a Frobenius-type triangular matrix algebra $\Lambda$ and the module category of $\Lambda$ are equivalent.

We denote $B_{j i}=\operatorname{Hom}_{A_{i}}\left(B_{i j}, A_{i}\right) \cong \operatorname{Hom}_{A_{j}}\left(B_{i j}, A_{j}\right)$ for $1 \leq i<j \leq n$.

Proposition 2.2. For $1 \leq i<j \leq n, B_{j i}$ is a free left $A_{j}$-module and a free right $A_{i}$-module. Also, we have $B_{i j}=\operatorname{Hom}_{A_{i}}\left(B_{j i}, A_{i}\right) \cong \operatorname{Hom}_{A_{j}}\left(B_{j i}, A_{j}\right)$.

Proof. It is trivial.

Proposition 2.3. For any $A_{j}$-module $M_{j}$ and $A_{i}$-module $N_{i}$, we have the isomorphism

$$
\operatorname{Hom}_{A_{i}}\left(B_{i j} \otimes M_{j}, N_{i}\right) \cong \operatorname{Hom}_{A_{j}}\left(M_{j}, B_{j i} \otimes N_{i}\right) .
$$

Proof. The adjunction map gives an isomorphism of $k$-vector spaces:

$$
\operatorname{Hom}_{A_{i}}\left(B_{i j} \otimes M_{j}, N_{i}\right) \cong \operatorname{Hom}_{A_{j}}\left(M_{j}, \operatorname{Hom}_{A_{i}}\left(B_{i j}, N_{i}\right)\right) .
$$

Since $\operatorname{Hom}_{A_{i}}\left(B_{i j}, N_{i}\right) \cong \operatorname{Hom}_{A_{i}}\left(B_{i j}, A_{i} \otimes_{A_{i}} N_{i}\right) \cong \operatorname{Hom}_{A_{i}}\left(B_{i j}, A_{i}\right) \otimes_{A_{i}} N_{i} \cong B_{j i} \otimes_{A_{i}} N_{i}$, we have that: $\operatorname{Hom}_{A_{i}}\left(B_{i j} \otimes M_{j}, N_{i}\right) \cong \operatorname{Hom}_{A_{j}}\left(M_{j}, B_{j i} \otimes N_{i}\right)$.

Lemma 2.4. [The Dual Basis Lemma] Let $A$ be an Artin algebra, and $P$ be a finite generated projective $A$-module, $\operatorname{Hom}_{A}(P, A)$, there exist $x_{1}, \cdots, x_{m} \in P, f_{1}, \cdots, f_{m} \in$ $\operatorname{Hom}_{A}(P, A)$, such that for each $x \in P, x=\sum_{i=1}^{m} f_{i}(x) x_{i}$.

Since $B_{i j}$ is finite generated projective left $A_{i}$-module and finite generated projective right $A_{j}$-module, by the above lemma, there exist $L_{i j} \subseteq B_{i j}, L_{i j}^{*} \subseteq \operatorname{Hom}_{A_{i}}\left(B_{i j}, A_{i}\right)$ and $R_{i j} \subseteq$ $B_{i j}, R_{i j}^{*} \subseteq \operatorname{Hom}_{A_{j}}\left(B_{i j}, A_{j}\right)$ such that for each $b_{i j} \in B_{i j}, b_{i j}=\Sigma_{\ell \in L_{i j}} \ell^{*}\left(b_{i j}\right) \ell=\Sigma_{r \in R_{i j}} r^{*}\left(b_{i j}\right) r$.

For the isomorphism in Proposition 2.3, for each $\phi_{i j} \in \operatorname{Hom}_{A_{i}}\left(B_{i j} \otimes M_{j}, N_{i}\right)$, we have $\overline{\phi_{i j}} \in \operatorname{Hom}_{A_{j}}\left(M_{j}, B_{j i} \otimes N_{i}\right)$ satisfying $\overline{\phi_{i j}}\left(m_{j}\right)=\Sigma_{\ell \in L_{i j}} \ell^{*} \otimes \phi_{i j}\left(\ell \otimes m_{j}\right)$.

On the other hand, for each $\psi_{i j} \in \operatorname{Hom}_{A_{j}}\left(M_{j}, B_{j i} \otimes N_{i}\right)$, we have $\overline{\psi_{i j}} \in \operatorname{Hom}_{A_{i}}\left(B_{i j} \otimes M_{j}, N_{i}\right)$ satisfying $\overline{\psi_{i j}}\left(b_{i j} \otimes m_{j}\right)=\Sigma_{\ell \in L_{i j}} \ell^{*}\left(b_{i j}\right) \psi_{i j}\left(m_{j}\right)_{\ell}$, where the elements $\psi_{i j}\left(m_{j}\right)_{l} \in N_{i}$ are uniquely determined by $\psi_{i j}\left(m_{j}\right)=\Sigma_{\ell \in L_{i j}} \ell^{*} \otimes \psi_{i j}\left(m_{j}\right)_{\ell}$.

Let $\Lambda$ be a Frobenius-type triangular matrix algebra as in Definition 1.1. Denote $P_{i}=\Lambda e_{i}$ the projective $\Lambda$-module for the idempotent $e_{i}$ as the unit 1 of $A_{i}$ and $I_{i}$ the corresponding 
injective $\Lambda$-module for $1 \leq i \leq n$. Obviously,

$$
P_{i}=\left(\begin{array}{llllll}
A_{1 i} & A_{2 i} & \cdots & A_{i i} & 0 & \cdots
\end{array}\right)^{t} .
$$

Also, we denote $E_{i}$ the $\Lambda$-module $\left(0 \cdots A_{i} \cdots 0\right)^{t}$ where $A_{i}$ is in the $i$ - th row.

Lemma 2.5. [16] For two k-algebras $A$ and $B$, assume that $M$ is an $A$-B-bimodule such that $M$ is a projective left $A$-module, $P$ is a projective left $B$-module. Then $M \otimes_{B} P$ is a projective left A-module.

Proposition 2.6. For every $i=1, \ldots, n, \operatorname{proj} \cdot \operatorname{dim}\left(E_{i}\right) \leq 1$ and $i n j \cdot \operatorname{dim}\left(E_{i}\right) \leq 1$.

Proof. Clearly, $E_{1}=P_{1}$. For every $i=2, \ldots, n$, we have exact sequences:

$$
0 \rightarrow \oplus_{j=1}^{i-1} P_{j} \otimes_{A_{j}} B_{j i} \rightarrow P_{i} \rightarrow E_{i} \rightarrow 0 .
$$

So, proj.dim $\left(E_{i}\right) \leq 1$ by Lemma 2.5. Let $E_{i}^{\prime}$ be the right $\Lambda$-module such that $D\left(E_{i}^{\prime}\right) \cong E_{i}$. For $i=1, \cdots, n-1$, there is a canonical exact sequence

$$
0 \rightarrow \oplus_{j=i+1}^{n} B_{i j} \otimes_{A_{j}} e_{j} \Lambda \rightarrow e_{i} \Lambda \rightarrow E_{i}^{\prime} \rightarrow 0 .
$$

Applying the duality $D$ to (5) we get a minimal injective resolution

$$
0 \rightarrow E_{i} \rightarrow D\left(e_{i} \Lambda\right) \rightarrow \oplus_{j=i+1}^{n} D\left(B_{i j} \otimes_{A_{j}} e_{j} \Lambda\right) \rightarrow 0 .
$$

Clearly, $E_{n}$ is injective. So, $\operatorname{inj} \operatorname{dim}\left(E_{i}\right) \leq 1$ for $1 \leq i \leq n$.

Definition 2.7. For a Frobenius-type triangular matrix algebra $\Lambda$, following [11], a finitely generated $\Lambda$-representation $X$ is called locally free if $X_{i}$ are free $A_{i}$-modules for all $1 \leq i \leq n$.

Denote by rep l.f. $_{(}(\Lambda)$ the subcategory of all locally free $\Lambda$-modules.

Corollary 2.8. For a Frobenius-type triangular matrix algebra $\Lambda$, if $X \in \operatorname{rep}_{\text {l.f. }}(\Lambda)$, then $\operatorname{proj} \cdot \operatorname{dim}(X) \leq 1, \operatorname{inj} \cdot \operatorname{dim}(X) \leq 1$.

Proof. We have a short exact sequence

$$
0 \rightarrow e_{1} X \rightarrow X \rightarrow\left(1-e_{1}\right) X \rightarrow 0 .
$$

where $e_{1} X$ and $\left(1-e_{1}\right) X$ are locally free. By Proposition 2.6 and using induction on $n$, we know that the projective and the injective dimensions of $e_{1} X$ and $\left(1-e_{1}\right) X$ are at most one.

Remark 2.9. For the algebra $A=A(C, D, \Omega)$ in [11], the three conditions

$$
X \in \operatorname{rep}_{\text {l.f. }}(A), \quad \operatorname{proj} \cdot \operatorname{dim}(X) \leq 1, \quad \operatorname{inj} \cdot \operatorname{dim}(X) \leq 1
$$

are equivalent. But it is generally not true for a Frobenius-type triangular matrix algebra. So, Corollary 2.8 is available for the sequel. 
An algebra $A$ is called $m$-Gorenstein if

$$
\operatorname{inj} \cdot \operatorname{dim}(A) \leq m \text { and } \operatorname{proj} \cdot \operatorname{dim}(D A) \leq m
$$

Such algebras were firstly introduced and studied in [12].

Corollary 2.10. The Frobenius-type triangular matrix algebra $\Lambda$ is a 1-Gorenstein algebra.

Proof. It is a direct consequence of Corollary 2.8 .

Recall that for an algebra $A$ an $A$-module $X$ is $\tau$-rigid (resp. $\tau^{-}$-rigid) if $\operatorname{Hom}_{A}(X, \tau(X))=$ 0 (resp. $\operatorname{Hom}_{A}\left(\tau^{-}(X), X\right)=0$ ). We call $\Lambda$-mod $X$ rigid if $\operatorname{Ext}_{\Lambda}^{1}(X, X)=0$.

When proj.dim $(X) \leq 1$, we have a functorial isomorphism $\operatorname{Ext}_{A}^{1}(X, Y) \cong \operatorname{DHom}_{A}(Y, \tau(X))$; when $i n j \operatorname{dim}(X) \leq 1$, we have a functorial isomorphism $\operatorname{Ext}_{A}^{1}(X, Y) \cong \operatorname{DHom}_{A}\left(\tau^{-}(Y), X\right)$.

Corollary 2.11. For a Frobenius-type triangular matrix algebra $\Lambda$, let $X \in$ rep $p_{\text {l.f. }}(\Lambda)$. Then, $X$ is rigid if and only if $X$ is $\tau$-rigid, also if and only if $X$ is $\tau^{-}$-rigid.

Proposition 2.12. For a Frobenius-type triangular matrix algebra $\Lambda$, the subcategory rep $p_{\text {l.f. }}(\Lambda)$ is closed under extensions, kernels of epimorphisms and cokernels of monomorphisms.

Proof. Let $0 \rightarrow X \stackrel{f}{\rightarrow} Y \stackrel{g}{\rightarrow} Z \rightarrow 0$ be a short exact sequence in $\operatorname{rep}(\Lambda)$. For each $1 \leq i \leq n$, this induces a short exact sequence

$$
0 \rightarrow e_{i} X \stackrel{f}{\rightarrow} e_{i} Y \stackrel{g}{\rightarrow} e_{i} Z \rightarrow 0
$$

of left $A_{i}$-modules.

By the definition, when $X, Z \in \operatorname{rep}_{\text {l.f. }}(\Lambda), e_{i} X, e_{i} Z$ are both $A_{i}$-free and then $e_{i} X$ is injective via $A_{i}$ is a Frobenius algebra and $e_{i} Z$ is projective for any $i$. Hence, the short exact sequence (7) splits, i.e. $e_{i} Y \cong e_{i} X \oplus e_{i} Z$. It follows that each $e_{i} Y$ is free $A_{i}$-module and then $Y \in \operatorname{rep}_{l . f .}(\Lambda)$.

When $Y, Z \in \operatorname{rep}_{\text {l.f. }}(\Lambda), e_{i} Y, e_{i} Z$ are both $A_{i}$-free and then $e_{i} Z$ is a projective module. Hence (7) splits, i.e. $e_{i} Y \cong e_{i} X \oplus e_{i} Z$. By the Krull-Schmidt theorem, it is easy to see that each $e_{i} X$ is a free $A_{i}$-module. So, $X \in \operatorname{rep}_{l . f .}(\Lambda)$.

When $X, Y \in \operatorname{rep}_{\text {l.f. }}(\Lambda), e_{i} X, e_{i} Y$ are both $A_{i}$-free and then $e_{i} X$ is injective via $A_{i}$ is a Frobenius algebra. Hence $(7)$ splits. Similarly, by the Krull-Schmidt theorem, each $e_{i} Z$ is free $A_{i}$-module. So, $Z \in \operatorname{rep}_{\text {l.f. }}(\Lambda)$. 


\section{Reflection Functors and AR-translation}

$$
\begin{aligned}
& \text { Let } \Lambda=\left(\begin{array}{cccc}
A_{1} & A_{12} & \ldots & A_{1 n} \\
0 & A_{2} & \ldots & A_{2 n} \\
\vdots & \vdots & \ddots & \vdots \\
0 & 0 & \cdots & A_{n}
\end{array}\right) \text { be a Frobenius-type triangular matrix algebra. Denote } \\
& S_{1}(\Lambda)=\left(\begin{array}{ccccc}
A_{2} & A_{23} & \cdots & A_{2 n} & A_{21} \\
0 & A_{3} & \ldots & A_{3 n} & A_{31} \\
\vdots & \vdots & \ddots & \vdots & \vdots \\
0 & 0 & \cdots & A_{n} & A_{n 1} \\
0 & 0 & \cdots & 0 & A_{1}
\end{array}\right) \text {, where } B_{j 1}=\operatorname{Hom}_{A_{1}}\left(B_{1 j}, A_{1}\right) \text { and } \\
& A_{j 1}= \oplus_{l=0}^{n-j} \oplus_{j<k_{1}<k_{2}<\cdots<k_{l} \leq n} B_{j k_{1}} \otimes_{A_{k_{1}}} B_{k_{1} k_{2}} \otimes \cdots \otimes_{A_{k_{l}}} B_{k_{l} 1}
\end{aligned}
$$

for $j=2, \ldots, n$.

$B_{j 1}$ is a free right $A_{1}$-module and also a free left $A_{j}$-module, since $B_{j 1}=\operatorname{Hom}_{A_{1}}\left(B_{1 j}, A_{1}\right) \cong$ $H_{o m} A_{j}\left(B_{1 j}, A_{j}\right)$. For $S_{1}(\Lambda)$, we have

$\operatorname{Hom}_{A_{1}}\left(B_{j 1}, A_{1}\right) \cong \operatorname{Hom}_{A_{1}}\left(\operatorname{Hom}_{A_{1}}\left(B_{1 j}, A_{1}\right), A_{1}\right) \cong B_{1 j} \cong \operatorname{Hom}_{A_{j}}\left(\operatorname{Hom}_{A_{j}}\left(B_{1 j}, A_{j}\right), A_{j}\right) \cong$ $\operatorname{Hom}_{A_{j}}\left(B_{j 1}, A_{j}\right)$,

which means that $S_{1}(\Lambda)$ is still a Frobenius-type triangular matrix algebra.

Using the same method in sequence, we can obtain the Frobenius-type triangular matrix algebra $S_{k} S_{k-1} \ldots S_{1}(\Lambda)$ for any $k$. In particular, it can be seen that $\Lambda \cong S_{n} S_{n-1} \ldots S_{1}(\Lambda)$.

A reflection functor $F_{1}^{+}: \operatorname{rep}(\Lambda) \rightarrow \operatorname{rep}\left(s_{1}(\Lambda)\right)$ can be described as follows.

$$
\begin{gathered}
\text { For } X=\left(\begin{array}{c}
X_{1} \\
X_{2} \\
\vdots \\
X_{n}
\end{array}\right)_{\phi_{i j}} \in \operatorname{rep}(\Lambda) \text {, define } F_{1}^{+}(X)=\left(\begin{array}{c}
X_{2} \\
\vdots \\
X_{n} \\
X_{1}^{\prime}
\end{array}\right)_{\phi_{i j}^{\prime}} \text {, with } X_{1}^{\prime}=\operatorname{Ker}\left(X_{1, i n}\right) \text {, where } \\
X_{1, i n}: \oplus_{k=2}^{n} B_{1 k} \otimes X_{k} \rightarrow X_{1}
\end{gathered}
$$

Denote by $\psi_{i 1}$ the composition of the inclusion map $X_{1}^{\prime} \hookrightarrow \oplus_{k=2}^{n} B_{1 k} \otimes X_{k}$ and the projection $\oplus_{k=2}^{n} B_{1 k} \otimes X_{k} \rightarrow B_{1 i} \otimes X_{i}$. Then

$$
\phi_{i j}^{\prime}= \begin{cases}\overline{\psi_{i 1}}, & \text { for } j=1 \\ \phi_{i j}, & \text { otherwise. }\end{cases}
$$


For a morphism $f=\left\{f_{i}\right\}: X \rightarrow Y$ in $\operatorname{rep}(\Lambda), F_{1}^{+}(f)=f^{\prime}=\left\{f_{i}^{\prime}\right\}$ where $f_{i}^{\prime}=f_{i}$ for $i=2, \ldots, n$ and $f_{1}^{\prime}$ is the unique morphism make the following diagram commutes:

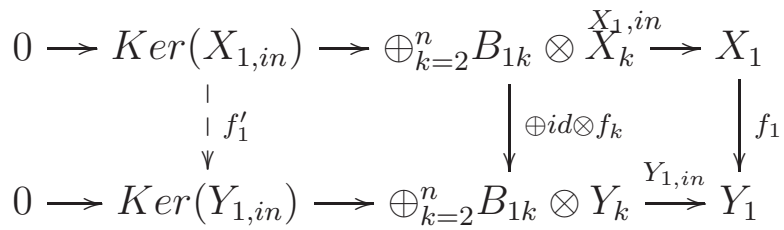

$$
\begin{aligned}
& \text { Similarly, for } X=\left(\begin{array}{c}
X_{2} \\
\vdots \\
X_{n} \\
X_{1}
\end{array}\right)_{\phi_{i j}} \in \operatorname{rep}\left(S_{1}(\Lambda)\right) \text {, define } F_{1}^{-}: \operatorname{rep}\left(S_{1}(\Lambda)\right) \rightarrow \operatorname{rep}(\Lambda) \text { satisfying } \\
& F_{1}^{-}(X)=\left(\begin{array}{c}
X_{1}^{\prime} \\
X_{2} \\
\vdots \\
X_{n}
\end{array}\right)_{\phi_{i j}^{\prime}} \text {, with } X_{1}^{\prime}=\operatorname{Coker}\left(X_{1, \text { out }}\right) \text {, where } X_{1, \text { out }}:=\left(\overline{\phi_{j 1}}\right)_{j}: X_{1} \rightarrow \oplus_{j=2}^{n} B_{1 j} \otimes X_{j} \text {. }
\end{aligned}
$$

Denote by $\psi_{1 j}$ the composition of the inclusion map $B_{1 j} \otimes X_{j} \hookrightarrow \oplus_{k=2}^{n} B_{1 k} \otimes X_{k}$ and the projection $\oplus_{k=2}^{n} B_{1 k} \otimes X_{k} \rightarrow X_{1}^{\prime}$. Then

$$
\phi_{i j}^{\prime}= \begin{cases}\psi_{1 j}, & \text { if } i=1 \\ \phi_{i j}, & \text { otherwise }\end{cases}
$$

For a morphism $g=\left\{g_{i}\right\}: X \rightarrow Y$ in $\operatorname{rep}\left(S_{1}(\Lambda)\right), F_{1}^{-}(g)=g^{\prime}=\left\{g_{i}^{\prime}\right\}$ where $g_{i}^{\prime}=g_{i}$ for $i=2, \ldots, n$ and $g_{1}^{\prime}$ is the unique morphism make the following diagram commutes:

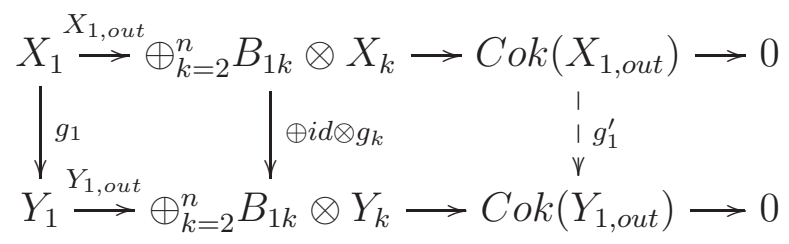

In the same way in sequence, for any $k$, we can define

$$
\begin{aligned}
& F_{k}^{+}: \operatorname{rep}\left(S_{k-1} S_{k-2} \ldots S_{1}(\Lambda)\right) \rightarrow \operatorname{rep}\left(S_{k} S_{k-1} \ldots S_{1}(\Lambda)\right), \\
& F_{k}^{-}: \operatorname{rep}\left(S_{k} S_{k-1} \ldots S_{1}(\Lambda)\right) \rightarrow \operatorname{rep}\left(S_{k-1} S_{k-2} \ldots S_{1}(\Lambda)\right) .
\end{aligned}
$$

Then we denote

$$
C^{+}=F_{n}^{+} F_{n-1}^{+} \ldots F_{1}^{+}: \operatorname{rep}(\Lambda) \rightarrow \operatorname{rep}(\Lambda) \text { and } C^{-}=F_{1}^{-} F_{2}^{-} \ldots F_{n}^{-}: \operatorname{rep}(\Lambda) \rightarrow \operatorname{rep}(\Lambda)
$$

which are called the Coxeter functors on $\operatorname{rep}(\Lambda)$. 
Remark 3.1. Let $Q$ be a connected acyclic quiver. We can re-arrange the vertices of $Q$ by making $i<j$ if there is a path from $j$ to $i$ so as to get an admissible sequence $1,2, \cdots, n$ with 1 as a sink vertex and $n$ as a source vertex. Thus, the path algebra $k Q$ can be seen as a special case of Frobenius-type triangular matrix algebras through assuming $A_{i}=k$ for all $i$ and $B_{i j}$ is the $k$-linear spaces generated by all arrows from $j$ to $i$. In this case, the reflection functors and Coxeter functors defined above accords with that as the classical case given by Dlab and Ringel in [6][7][18].

Proposition 3.2. For a Frobenius-type triangular matrix algebra $\Lambda$, and $X \in \operatorname{rep}\left(S_{1}(\Lambda)\right), Y \in$ $\operatorname{rep}(\Lambda)$, there is a functorial isomorphism

$$
\operatorname{Hom}_{\Lambda}\left(F_{1}^{-}(X), Y\right) \cong \operatorname{Hom}_{S_{1}(\Lambda)}\left(X, F_{1}^{+}(Y)\right) .
$$

Proof. Consider a morphism $f \in \operatorname{Hom}_{S_{1}(\Lambda)}\left(X, F_{1}^{+}(Y)\right)$. By definition, this is a collection of $A_{j}$-module homomorphisms $f_{j}: X_{j} \rightarrow\left(F_{1}^{+}(Y)\right)_{j}$ with $1 \leq j \leq n$ satisfying certain commutative relations. In the diagram

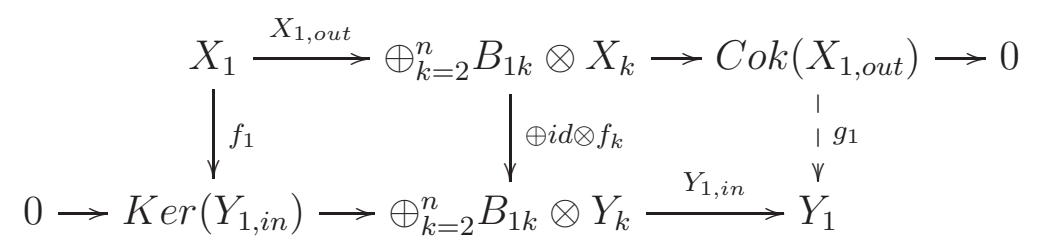

its left square commutes. Observe that $Y_{1, \text { in }} \circ\left(\oplus i d \otimes f_{k}\right) \circ X_{1, \text { out }}=0$, so $Y_{1, \text { in }} \circ\left(\oplus i d \otimes f_{k}\right)$ factors through the cokernel of $X_{1, \text { out }}$. Then, there is a map $g_{1}$ such that the right square commutes.

Thus if we set $g_{j}:=f_{j}$ for $2 \leq j \leq n$, we get a homomorphism $g: F_{1}^{-}(X) \rightarrow Y$ corresponding to the given $f$. Write $\pi(f)=g$.

Conversely, consider a homomorphism $g: F_{1}^{-}(X) \rightarrow Y$, in the diagram

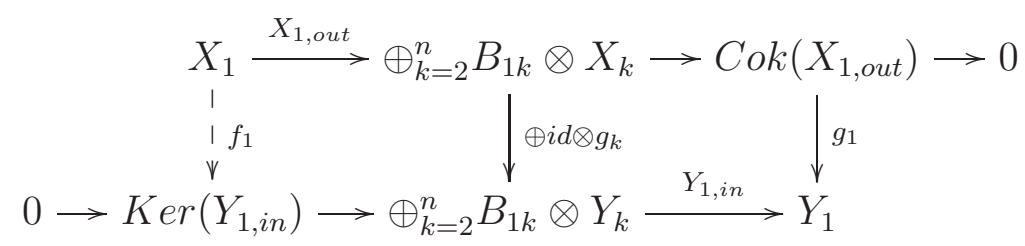

the right square commutes. There is a unique map $f_{1}$ such that the left square commutes.

Similarly as above, let $f_{j}:=g_{j}$ for $2 \leq j \leq n$ then we get a homomorphism $f: F_{1}^{-}(X) \rightarrow Y$ corresponding to the given $g$. Write $\tau(g)=f$.

For a morphism $f \in \operatorname{Hom}_{S_{1}(\Lambda)}\left(X, F_{1}^{+}(Y)\right)$, there exists a unique $\pi(f)$ makes the right square above commutes. And for $\pi(f)$, there exists a unique $\tau \pi(f)$ makes the left square above commutes. Since $f$ makes the left square above commutes, we have $\tau \pi(f)=f$. Similarly, $\pi \tau(g)=g$. 
Therefore, $\pi$ and $\tau$ are mutual-inverse, and then we get a functorial isomorphism

$$
\operatorname{Hom}_{\Lambda}\left(F_{1}^{-}(X), Y\right) \cong \operatorname{Hom}_{S_{1}(\Lambda)}\left(X, F_{1}^{+}(Y)\right) .
$$

Lemma 3.3. For a Frobenius-type triangular matrix algebra $\Lambda$, there is a short exact sequence of $\Lambda$ - $\Lambda$-bimodules

$$
P_{\bullet}: 0 \rightarrow \oplus_{1 \leq i<j \leq n} \Lambda e_{i} \otimes_{A_{i}} B_{i j} \otimes_{A_{j}} e_{j} \Lambda \stackrel{d}{\rightarrow} \oplus_{k=1}^{n} \Lambda e_{k} \otimes_{A_{k}} e_{k} \Lambda \stackrel{\text { mult }}{\rightarrow} \Lambda \rightarrow 0
$$

where $d$ satisfies $d\left(p \otimes_{A_{i}} b \otimes_{A_{j}} q\right):=p b \otimes_{A_{j}} q-p \otimes_{A_{i}} b q$ and the morphism "mult" is given by the multiplication of $\Lambda$.

Proof. Trivially, $d$ is injective and mult is surjective. Also, $\operatorname{Im}(d)=\operatorname{Ker}($ mult $)$.

Corollary 3.4. For a Frobenius-type triangular matrix algebra $\Lambda$ and $X \in$ rep $_{\text {l.f. }}(\Lambda)$, there is a projective resolution of $X$ :

$$
P . \otimes_{\Lambda} X: 0 \rightarrow \oplus_{1 \leq i<j \leq n} P_{i} \otimes_{A_{i}} B_{i j} \otimes_{A_{j}} X_{j} \stackrel{d \otimes X}{\rightarrow} \oplus_{k=1}^{n} P_{k} \otimes_{A_{k}} X_{k} \stackrel{\text { mult }}{\rightarrow} X \rightarrow 0
$$

with $(d \otimes X)\left(p \otimes_{A_{i}} b \otimes_{A_{j}} x\right)=p b \otimes_{A_{j}} x-p \otimes_{A_{i}} \phi_{i j}\left(b \otimes_{A_{j}} x\right)$.

Proof. Here $P_{i}$ is just defined in (3). Then, $P_{\bullet} \otimes_{\Lambda} X$ is always exact. Since $X$ is locally free, $e_{k} \Lambda \otimes_{\Lambda} X=e_{k} X$ are free $A_{k}$-modules. Thus $P_{\bullet} \otimes_{\Lambda} X$ is a projective resolution.

Following [10] and [11], we define a functor $T$ of $\operatorname{rep}(\Lambda)$ satisfying that

$$
T X=\left(\begin{array}{c}
X_{1} \\
X_{2} \\
\vdots \\
X_{n}
\end{array}\right)_{\psi_{i j}} \quad \text { for any } X=\left(\begin{array}{c}
X_{1} \\
X_{2} \\
\vdots \\
X_{n}
\end{array}\right)_{\phi_{i j}} \in \operatorname{rep}(\Lambda)
$$

where $\psi_{i j}\left(b \otimes_{j} x\right)=-\phi_{i j}\left(b \otimes_{j} x\right)$ for $b \in B_{i j}, x \in X_{j}, 1 \leq i<j \leq n$. Obviously, $T$ is an automorphism functor.

Theorem 3.5. For a Frobenius-type triangular matrix algebra $\Lambda$ and each $X \in$ rep $_{\text {l.f. }}(\Lambda)$, there are functorial isomorphisms:

$$
T C^{+}(X) \cong \tau(X) \text { and } T C^{-}(X) \cong \tau^{-}(X) .
$$

This theorem and its preparation above follow the conclusion and method of [10] and [11]. But, here $\Lambda$ is a Frobenius-type triangular matrix algebra, which includes the classes of algebras in [10] and [11]. We need to overcome the different key point such as the different expression form of algebras by using the dual basis in Lemma 2.4 .

Firstly, from $\Lambda$, we construct a new Frobenius-type triangular matrix algebra: 


$$
\tilde{\Lambda}=\left(\begin{array}{cccc}
\tilde{A}_{1} & \tilde{A}_{1,2} & \ldots & \tilde{A}_{1,2 n} \\
0 & \tilde{A}_{2} & \ldots & \tilde{A}_{2,2 n} \\
\vdots & \vdots & \ddots & \vdots \\
0 & 0 & \ldots & \tilde{A}_{2 n}
\end{array}\right) \text {, where } \tilde{A}_{i}= \begin{cases}A_{i}, & \text { if } 1 \leq i \leq n, \\
A_{i-n}, & \text { if } n+1 \leq i \leq 2 n,\end{cases}
$$

and $\quad \tilde{B}_{i j}= \begin{cases}B_{i j}, & \text { if } 1 \leq i<j \leq n \text { or } n+1 \leq i<j \leq 2 n \\ \operatorname{Hom}_{A_{i}}\left(B_{j-n, i}, A_{i}\right), & \text { if } 1 \leq j-n<i \leq n \\ 0, & \text { otherwise }\end{cases}$

and $\tilde{A}_{i j}=\oplus_{l=0}^{j-i-1} \oplus_{i<k_{1}<k_{2}<\cdots<k_{l}<j} \tilde{B}_{i k_{1}} \otimes_{\tilde{A}_{k_{1}}} \tilde{B}_{k_{1} k_{2}} \otimes \cdots \otimes_{\tilde{A}_{k_{l}}} \tilde{B}_{k_{l} j}$ for $1 \leq i<j \leq 2 n$.

For any non-negative integer $t$, denote

$$
1^{(t)}:=\sum_{i=t+1}^{t+n} e_{i}, 1_{0}^{(t)}:=\Sigma_{i=1}^{t+n} e_{i}
$$

and the corresponding subalgebras

$$
\Lambda^{(t)}:=1^{(t)} \tilde{\Lambda} 1^{(t)}, \tilde{\Lambda}^{(t)}:=1_{0}^{(t)} \tilde{\Lambda} 1_{0}^{(t)} .
$$

for $0 \leq t \leq n$. It is easy to see that $\tilde{\Lambda}^{(0)} \cong \Lambda^{(0)} \cong \Lambda^{(n)} \cong \Lambda, \tilde{\Lambda}^{(n)} \cong \tilde{\Lambda}$. And $\Lambda^{(t)} \cong S_{t} \cdots S_{1}(\Lambda)$ for $1 \leq t \leq n$.

Let $X=\left(\begin{array}{c}X_{1} \\ \vdots \\ X_{2 n}\end{array}\right)_{\phi_{i j}} \in \operatorname{rep}(\tilde{\Lambda})$ satisfy the following condition:

$$
X_{n+i} \stackrel{\tilde{X}_{i, o u t}}{\longrightarrow} \oplus_{k=i+1}^{n+i-1} B_{i k} \otimes X_{k} \stackrel{\tilde{X}_{i, i n}}{\longrightarrow} X_{i} \text { such that } \tilde{X}_{i, i n} \circ \tilde{X}_{i, \text { out }}=0 \text { for } 1 \leq i \leq n .
$$

We define the restriction functors:

$$
\begin{gathered}
\operatorname{Res}{ }^{(t, m)}: \operatorname{rep}\left(\tilde{\Lambda}^{(m)}\right) \rightarrow \operatorname{rep}\left(\Lambda^{(t)}\right), X \mapsto 1^{(t)} \tilde{\Lambda}^{(m)} \otimes_{\tilde{\Lambda}^{(m)}} X . \\
\operatorname{Res}_{(t, m)}: \operatorname{rep}\left(\tilde{\Lambda}^{(m)}\right) \rightarrow \operatorname{rep}\left(\tilde{\Lambda}^{(t)}\right), X \mapsto 1_{0}^{(t)} \tilde{\Lambda}^{(m)} \otimes_{\tilde{\Lambda}^{(m)}} X \text { for } 1 \leq t \leq m \leq n .
\end{gathered}
$$

Obviously, $\operatorname{Res}_{(t, m)}$ has a right adjoint

$$
\operatorname{Res}_{(t, m)}^{*}(-)=\operatorname{Hom}_{\tilde{\Lambda}^{(t)}}\left(1_{0}^{(t)} \tilde{\Lambda}^{(m)},-\right) .
$$

The following lemma is a generalization of [11, Lemma 10.2]. Their proofs are identical.

Lemma 3.6. With the above notations, there is a functorial isomorphisms

$$
\operatorname{Res}^{(i, i)} \circ \operatorname{Res}_{(i-1, i)}^{*}(X) \cong F_{i}^{+} \circ \operatorname{Res}^{(i-1, i-1)}(X)
$$

for $X \in \operatorname{rep}\left(\tilde{\Lambda}^{(i-1)}\right)$ which satisfies (8) for all $1 \leq i \leq n$. 
By Lemma 3.6 we obtain that for any $X \in \operatorname{rep}(\Lambda)$ (regarded as a representation of $\left.\operatorname{rep}\left(\tilde{\Lambda}^{(0)}\right)\right)$ we have

$$
\begin{aligned}
\operatorname{Hom}_{\Lambda}\left(1^{(0)} \tilde{\Lambda} 1^{(n)}, X\right) & =\operatorname{Res}^{(n, n)} \circ \operatorname{Res}_{(n-1, n)}^{*} \circ \cdots \circ \operatorname{Res}_{(0,1)}^{*}(X) \\
& =F_{n}^{+} \circ \operatorname{Res}^{n-1} \circ \operatorname{Res}_{(n-2, n-1)}^{*} \circ \cdots \circ \operatorname{Res}_{(0,1)}^{*}(X) \\
& =\cdots \\
& =F_{n}^{+} \circ F_{n-1}^{+} \circ \cdots F_{1}^{+} \circ \operatorname{Res}^{0}(X) \\
& =C^{+}(X) .
\end{aligned}
$$

Denote

$$
\operatorname{Res}_{0}=\operatorname{Res}_{(0,1)} \circ \operatorname{Res}_{(1,2)} \circ \cdots \circ \operatorname{Res}_{(n-1, n)}, \quad \operatorname{Res}_{0}^{*}=\operatorname{Res}_{(n-1, n)}^{*} \circ \cdots \circ \operatorname{Res}_{(1,2)}^{*} \circ \operatorname{Res}_{(0,1)}^{*} .
$$

It is easy to see that $R e s_{0}^{*}$ is right adjoint of $R e s_{0}$.

Now, following [10] and [11], we construct another functor $R_{0}^{*}: \operatorname{rep}\left(\Lambda^{(0)}\right) \rightarrow \operatorname{rep}(\tilde{\Lambda})$, and then show that $R_{0}^{*}$ is right adjoint to $\operatorname{Res}_{0}$.

Let $X \in \operatorname{rep}\left(\Lambda^{(0)}\right)$. We first define $\tilde{X} \in \operatorname{rep}(\tilde{\Lambda})$ by requiring that

$$
\begin{aligned}
\operatorname{Res}^{(0, n)}(\tilde{X}) & =X . \\
\operatorname{Res}^{(n, n)}(\tilde{X}) & =\oplus_{1 \leq k<t \leq n} \operatorname{Hom}_{A_{t}}\left(\tilde{B}_{t, n+k} \otimes e_{n+k} \Lambda^{(n)}, X_{t}\right) .
\end{aligned}
$$

For $1 \leq i<j \leq n$, it remains to define the structure map of $\tilde{X}$ as $A_{j}$-module morphisms:

$$
\phi_{j, n+i}: \tilde{B}_{j, n+i} \otimes \tilde{X}_{n+i} \rightarrow \tilde{X}_{j}=X_{j}
$$

which is given by the following composition:

$$
\begin{aligned}
& \tilde{B}_{j, n+i} \otimes_{A_{i}}\left(\oplus_{1 \leq k<t \leq n} \operatorname{Hom}_{A_{t}}\left(\tilde{B}_{t, n+k} \otimes e_{n+k} \Lambda^{(n)} e_{n+i}, X_{t}\right)\right) \\
& \stackrel{\text { proj. }}{\longrightarrow} \tilde{B}_{j, n+i} \otimes_{A_{i}} \operatorname{Hom}_{A_{j}}\left(\tilde{B}_{j, n+i} \otimes e_{n+i} \Lambda^{(n)} e_{n+i}, X_{j}\right) \\
& =\tilde{B}_{j, n+i} \otimes_{A_{i}} \operatorname{Hom}_{A_{j}}\left(\tilde{B}_{j, n+i}, X_{j}\right) \\
& \stackrel{\text { eval. }}{\longrightarrow} X_{j}
\end{aligned}
$$

where the first map is the projection on the direct summand indexed by $i, j$ and the second map is the evaluation $b \otimes \varphi=\varphi(b)$.

Secondly, we define a $\tilde{\Lambda}$-subrepresentation $R_{0}^{*}(X)$ of $\tilde{X}$ as follows. We set

$$
\left(R_{0}^{*}(X)\right)_{i}=\tilde{X}_{i}=X_{i}, \quad(1 \leq i \leq n),
$$

and we define $R_{0}^{*}(X)_{n+i}$ as the subspace of $\tilde{X}_{n+i}$ generated by all of

$$
\left(\mu_{k, t}^{i}\right)_{1 \leq k<t \leq n} \in \oplus_{1 \leq k<t \leq n} \operatorname{Hom}_{A_{t}}\left(\tilde{B}_{t, n+k} \otimes e_{n+k} \Lambda^{(n)} e_{n+i}, X_{t}\right)
$$

such that, for all $1 \leq t \leq n$ and $\lambda \in e_{n+t} \Lambda^{(n)} e_{n+i}$ the following relation holds:

$$
\Sigma_{1 \leq k<t, \ell \in L_{k t}} \mu_{k, t}^{i}\left(\ell^{*} \otimes \ell \lambda\right)+\Sigma_{t<m \leq n, r \in R_{t m}} \phi_{t m}\left(r \otimes \mu_{t, m}^{i}\left(r^{*} \otimes \lambda\right)\right)=0 .
$$


For $\mu^{j}=\left(\mu_{k, t}^{j}\right)_{1 \leq k<t \leq n} \in\left(R_{0}^{*}(X)\right)_{n+j}, 1 \leq j \leq n$, we deduce from the definitions that

$$
\begin{aligned}
& \tilde{X}_{j, \text { in }} \circ \tilde{X}_{j, \text { out }}\left(\mu^{j}\right) \\
= & \tilde{X}_{j, \text { in }}\left(\Sigma_{1 \leq i<j, \ell \in L_{i j}}\left(\ell^{*} \otimes \phi_{n+i, n+j}\left(\ell \otimes \mu^{j}\right)\right)+\Sigma_{j<k \leq n, r \in R_{i j}}\left(r \otimes \phi_{k, n+j}\left(r^{*} \otimes \mu^{j}\right)\right)\right) \\
= & \Sigma_{1 \leq i<j, \ell \in L_{i j}} \phi_{j, n+i}\left(\ell^{*} \otimes \phi_{n+i, n+j}\left(\ell \otimes \mu^{j}\right)\right)+\Sigma_{j<k \leq n, r \in R_{i j}} \phi_{j, k}\left(r \otimes \phi_{k, n+j}\left(r^{*} \otimes \mu^{j}\right)\right) \\
= & \Sigma_{1 \leq i<j, \ell \in L_{i j}} \mu_{i, j}^{j}\left(\ell^{*} \otimes \ell \otimes e_{n+j}\right)+\Sigma_{j<k \leq n, r \in R_{i j}} \phi_{j, k}\left(r \otimes \mu_{j, k}^{j}\left(r^{*} \otimes e_{n+j}\right)\right)=0
\end{aligned} .
$$

So, $R_{0}^{*}(X)$ satisfies $(8)$.

Thus, we have obtained a functor $R_{0}^{*}: \operatorname{rep}\left(\Lambda^{(0)}\right) \rightarrow \operatorname{rep}(\tilde{\Lambda}) ; X \mapsto R_{0}^{*}(X)$. We have that $R_{0}^{*}$ is isomorphic to $R e s_{0}^{*}$. This is according to the uniqueness of adjoint functors up to natural equivalence and the following lemma.

Lemma 3.7. $R_{0}^{*}$ is right adjoint to Res $_{0}$.

It is a generalization of [11, Lemma 10.3]. Their proofs are identical.

Lemma 3.8. Let $A_{i}$ be a Frobenius algebra, $U$ and $V$ be finite generated free $A_{i}$-modules, then we have an isomorphism $\operatorname{DHom}_{A_{i}}(U, V) \cong \operatorname{Hom}_{A_{i}}(V, U)$, where $D:=\operatorname{Hom}_{k}(-, k)$.

Proof. Since $A_{i}$ is a Frobenius algebra, $D A_{i} \cong A_{i}$. Let $U=\oplus_{j=1}^{n} A_{i}$ and $V=\oplus_{t=1}^{m} A_{i}$, then $D \operatorname{Hom}_{A_{i}}(U, V) \cong \oplus_{j=1}^{n} \oplus_{t=1}^{m} \operatorname{DHom}_{A_{i}}\left(A_{i}, A_{i}\right) \cong \oplus_{j=1}^{n} \oplus_{t=1}^{m} \operatorname{Hom}_{A_{i}}\left(A_{i}, A_{i}\right) \cong \operatorname{Hom}_{A_{i}}(V, U)$.

Proposition 3.9. For a Frobenius-type triangular matrix algebra $\Lambda$ and $X \in \operatorname{rep}_{\text {l.f. }}(\Lambda)$, there is an isomorphism

$$
\tau(T M) \cong \operatorname{Res}^{(n, n)} \circ R_{0}^{*}(X)
$$

where $\Lambda$ is identified with $\Lambda^{(0)}$ and $\Lambda^{(n)}$ by their definitions.

Proof. The proof is similar to [11, Proposition 10.4]. The only illustration we need to add is the fact that for a locally free $\Lambda$-module $X$, we have $\operatorname{DHom}_{A_{i}}\left(e_{i} \Lambda, X_{i}\right) \cong \operatorname{Hom}_{A_{i}}\left(X_{i}, e_{i} \Lambda\right)$. This follows from Lemma 3.8 .

Proof of Theorem 3.5: By Proposition 3.9, if $X \in \operatorname{rep}_{l . f .}(\Lambda)$ we have

$$
T C^{+}(X) \cong \operatorname{Res}^{(n, n)} \circ \operatorname{Res}_{0}^{*}(T X) \cong \operatorname{Res}^{(n, n)} \circ R_{0}^{*}(T X) \cong \tau\left(T^{2} X\right) \cong \tau(X) .
$$

Let $X, Y \in \operatorname{rep}_{l . f .}(\Lambda)$, then we have

$$
\begin{aligned}
\operatorname{Hom}_{\Lambda}\left(\tau^{-}(X), Y\right) & \cong \operatorname{Hom}_{\Lambda}(X, \tau(Y)) \\
& \cong \operatorname{Hom}_{\Lambda}\left(X, C^{+}(T Y)\right) \\
& \cong \operatorname{Hom}_{\Lambda}\left(C^{-}(T X), Y\right)
\end{aligned}
$$

The first isomorphism is obtained by Corollary 2.8 and the third isomorphism follows from Proposition 3.2. 
There exists a functorial isomorphism of right $\Lambda$-modules $D X \cong \operatorname{Hom}_{\Lambda}(X, D \Lambda)$ for all $\Lambda$-modules $X$. Since $D \Lambda \in \operatorname{rep}_{l . f .}(\Lambda)$, taking $Y=D \Lambda$ we get $\tau^{-}(X) \cong C^{-}(T X)$.

Recall that the category of Gorenstein-projective modules of $\Lambda$ is

$$
\mathcal{G P}(\Lambda)=\left\{X \in \operatorname{rep}(\Lambda) \mid \operatorname{Ext}_{\Lambda}^{1}(X, \Lambda)\right\}=0 .
$$

As an immediate consequence of Theorem 3.5 and the definition of $C^{+}(-)$we get the following result.

Corollary 3.10. For a Frobenius-type triangular matrix algebra $\Lambda$ and $X \in$ rep $_{\text {l.f. }}(\Lambda)$, the following are equivalent:

(i) $X \in \mathcal{G} \mathcal{P}(\Lambda)$;

(ii) $C^{+}(X)=0$;

(iii) $X_{i, \text { in }}$ is injective for all $1 \leq i \leq n$.

This result has been proved in a more general case in [16].

\section{Root Systems In CASE OF DYNKIN TYPE}

For a Frobenius-type triangular matrix algebra $\Lambda$, let $C=\left(c_{i j}\right) \in M_{n}(\mathbb{Z})$, where

$$
c_{i j}= \begin{cases}2, & \text { if } i=j \\ -\operatorname{rank}_{A_{i}}\left(B_{i j}\right) & \text { if } i<j \\ -\operatorname{rank}_{A_{i}}\left(B_{j i}\right) & \text { if } i>j .\end{cases}
$$

Denote $c_{i}=\operatorname{dim}_{k}\left(A_{i}\right)$, then it is easy to get that $c_{i} c_{i j}=c_{j} c_{j i}=-\operatorname{dim}_{k}\left(B_{i j}\right)$, which means that $C$ is a symmetrizable Cartan matrix.

Define a quadratic form $q_{C}: \mathbb{Z}^{n} \rightarrow \mathbb{Z}$ of $C$ satisfying for $x=\left(x_{1}, \cdots, x_{n}\right)^{t} \in \mathbb{Z}^{n}$,

$$
q_{C}(x):=\sum_{i=1}^{n} c_{i} x_{i}^{2}-\sum_{i<j} c_{i}\left|c_{i j}\right| x_{i} x_{j} .
$$

The Cartan matrix $C$ is said to be of Dynkin type (resp. Euclidean type) if $q_{C}$ is positive define (resp. positive semidefinite).

We define the valued quiver $\Gamma(\Lambda)$ via the Cartan matrix $C$ whose vertices are $1, \ldots, n$ and whose arrow $i \leftarrow j$ from $j$ to $i$ exists for each pair $(i, j)$ with $i<j$ if $c_{i j}<0$, with valuation $\left(-c_{j i},-c_{i j}\right)$ on the arrow $i \leftarrow j$. It is well-known, , see [13, Theorem 4.8], that

Fact 4.1. $C$ is of Dynkin type if and only if $\Gamma(\Lambda)$ is a disjoint union of quivers whose underlying valued graph is of Dynkin type.

The standard basis vector of $\mathbb{Z}^{n}$, denoted as $\alpha_{1}, \cdots, \alpha_{n}$, are the positive simple roots of the Kac-Moody algebra $\mathfrak{g}(C)$ associated with $C$, that is, of the quadratic form $q_{C}(x)$. For $1 \leq i, j \leq n$, define the reflections $s_{i}$ satisfying that $s_{i}\left(\alpha_{j}\right):=\alpha_{j}-c_{i j} \alpha_{i}$. 
The Weyl group $W(C)$ of $\mathfrak{g}(C)$ is the subgroup of $A u t\left(\mathbb{Z}^{n}\right)$ generated by $s_{1}, \cdots, s_{n}$. It is well-known that $W(C)$ is finite if and only if $C$ is of Dynkin type.

Let $\Delta(C)$ be the sets of roots of $C$ and $\Delta_{r e}(C):=\cup_{i=1}^{n} W\left(\alpha_{i}\right)$ be the set of real roots of $C$. Let $\Delta^{+}(C):=\Delta(C) \cap \mathbb{N}^{n}$ and $\Delta_{r e}^{+}(C):=\Delta_{r e}(C) \cap \mathbb{N}^{n}$. The following are equivalent:

(i) $C$ is of Dynkin type;

(ii) $\Delta(C)$ is finite;

(iii) $\Delta_{r e}(C)=\Delta(C)$.

Let

$$
\beta_{k}= \begin{cases}\alpha_{1}, & \text { if } k=1 \\ s_{1} s_{2} \cdots s_{k-1}\left(\alpha_{k}\right) & \text { if } 2 \leq k \leq n\end{cases}
$$

and

$$
\gamma_{k}= \begin{cases}s_{n} s_{n-1} \cdots s_{k+1}\left(\alpha_{k}\right) & \text { if } 1 \leq k \leq n-1, \\ \alpha_{n}, & \text { if } k=n .\end{cases}
$$

Let $c^{+}=s_{n} s_{n-1} \cdots s_{1}: \mathbb{Z}^{n} \rightarrow \mathbb{Z}^{n}$ and $c^{-}=s_{1} s_{2} \cdots s_{n}: \mathbb{Z}^{n} \rightarrow \mathbb{Z}^{n}$ be the Coxeter transformations. For $k \in \mathbb{Z}$, set $c^{k}:=\left\{\begin{array}{ll}\left(c^{+}\right)^{k} & \text { if } k>0, \\ \left(c^{-}\right)^{-k} & \text { if } k<0, \\ i d & \text { if } k=0 .\end{array}\right.$ It follows that $c^{+}\left(\beta_{k}\right)=-\gamma_{k}$.

The following three lemmas are well-known, for example see [3, Chapter VII].

Lemma 4.2. Suppose $C$ is not of Dynkin type. Then the element $c^{-r}\left(\beta_{i}\right)$ and $c^{s}\left(\gamma_{j}\right)$ with $r, s \geq 0$ and $1 \leq i, j \leq n$ are pairwise different elements in $\Delta_{r e}^{+}(C)$.

Let $C$ be of Dynkin type. Let $p_{i} \geq 1$ be minimal with $c^{-p_{i}}\left(\beta_{i}\right) \notin \mathbb{N}^{n}$ for $1 \leq i \leq n$, and let $q_{j} \geq 1$ be minimal with $c^{q_{j}}\left(\gamma_{j}\right) \notin \mathbb{N}^{n}$ for $1 \leq j \leq n$. The elements $c^{-r}\left(\beta_{i}\right)$ with $1 \leq i \leq n$ and $0 \leq r \leq p_{i}-1$ are pairwise different, and the elements $c^{s}\left(\gamma_{j}\right)$ with $1 \leq j \leq n$ and $0 \leq s \leq q_{j}-1$ are pairwise different.

Lemma 4.3. Assume that $C$ is of Dynkin type, then $\Delta^{+}(C)=\left\{c^{-r}\left(\beta_{i}\right) \mid 1 \leq i \leq n, 0 \leq r \leq\right.$ $\left.p_{i}-1\right\}=\left\{c^{s}\left(\gamma_{j}\right) \mid 1 \leq j \leq n, 0 \leq s \leq q_{j}-1\right\}$.

Lemma 4.4. Assume that $C$ is of Dynkin type. For every positive vector $\underline{x}$, there exist $s \geq 0$ such that $c^{s} \underline{x}>0$ but $c^{s+1} \underline{x} \ngtr 0$, and $t \geq 0$ such that $c^{-t} \underline{x}>0$ but $c^{-t-1} \underline{x} \ngtr 0$.

Definition 4.5. For a Frobenius-type triangular matrix algebra $\Lambda$, let $X$ be a locally free $\Lambda$-module. Let $r_{i}$ be the rank of the free $A_{i}$-module $X_{i}$. Following [11] we denote

$$
\underline{\operatorname{rank}}(X):=\left(r_{1}, \cdots, r_{n}\right) .
$$

Lemma 4.6. $\underline{\operatorname{rank}}\left(P_{k}\right)=\beta_{k}, \underline{\operatorname{rank}}\left(I_{k}\right)=\gamma_{k}$.

This lemma is a generalization of [11, Lemma 3.2 and 3.3]. Its proof is similar to the proof of [11, Lemma 3.2], using of the resolution (4) and (6). 
Definition 4.7. For a Frobenius-type triangular matrix algebra $\Lambda$, following [11], a $\Lambda$-module $X$ is called $X \tau$-locally free if $\tau^{k}(X)$ is locally free for all $k \in \mathbb{Z}$. Moreover, $X$ is called indecomposable $\tau$-locally free if $X$ cannot be written as a sum of two proper $\tau$-locally free $\Lambda$-modules.

The following proposition is a generalization of [11, Proposition 9.6 and 11.4].

Proposition 4.8. For a Frobenius-type triangular matrix algebra $\Lambda$, let $X$ be a rigid and locally free $\Lambda$-module,

(i) $F_{1}^{+}(X)$ is a rigid and locally free $S_{1}(\Lambda)$-module and $F_{n}^{-}$is a rigid and locally free $S_{n-i} \cdots S_{1}(\Lambda)$ module;

(ii) $X$ is $\tau$-locally free and $\tau^{k}(X)$ is rigid for all $k \in \mathbb{Z}$.

Proof. Its proof is identical to the proof of [11, Proposition 9.6 and 11.4]. The only illustration we need to add is the fact that for a locally free $\Lambda$-module $X$ and its minimal projective resolution of the form

$$
0 \rightarrow P^{\prime \prime} \rightarrow P^{\prime} \rightarrow X \rightarrow 0,
$$

we have that $P^{\prime}$ and $P^{\prime \prime}$ are two direct sums of $P_{i}$ for $1 \leq i \leq n$. This follows from Proposition 2.6, Corollary 2.8 and the horseshoe lemma.

Lemma 4.9. For a Frobenius-type triangular matrix algebra $\Lambda$, let $X$ be an indecomposable $\tau$ locally free $\Lambda$-module. Then $X$ is isomorphic to $E_{1}$ if and only if $F_{1}^{+}(X)=0$ (or equivalently, $\left.s_{1}(\underline{\operatorname{rank}}(X)) \ngtr 0\right)$. If $X \supsetneqq E_{1}$, then $F_{1}^{+}(X)$ is an indecomposable $\tau$-locally free $S_{1}(\Lambda)$-module and $\underline{\operatorname{rank}}\left(F_{1}^{+}(X)\right)=s_{1}(\underline{\operatorname{rank}}(X))$.

Proof. Since $X$ is indecomposable $\tau$-locally free, we obtain that $X_{1, i n}$ is surjective. If $X \nsubseteq E_{1}$, we have an exact sequence

$$
0 \rightarrow \operatorname{Ker}\left(X_{1, \text { in }}\right) \rightarrow \oplus_{k=2}^{n} B_{1 k} \otimes X_{k} \rightarrow X_{1} \rightarrow 0
$$

So

$$
\left.\underline{\operatorname{rank}}\left(F_{1}^{+}(X)\right)\right)_{1}=\sum_{k=2}^{n}\left|c_{1 k}\right| a_{k}-a_{1}=\left(s_{1}(\underline{\operatorname{rank}}(X))\right)_{1} .
$$

If $X \cong E_{1}$, we have $F_{1}^{+}(X)=0$ by the definition of $F_{1}^{+}$. And $s_{1}\left(\underline{\operatorname{rank}}\left(E_{1}\right)\right)_{1}=-1$.

We need the following two propositions, which are generalizations of $[11$, Proposition 11.5 and 11.6] respectively. Their proofs are similar.

Proposition 4.10. For a Frobenius-type triangular matrix algebra $\Lambda$ and a indecomposable $\tau$-locally free $\Lambda$-module $X$, the following statements hold:

(i) If $\tau^{k}(X) \neq 0$ for some $k \in \mathbb{Z}$, then $\underline{\operatorname{rank}}\left(\tau^{k}(X)\right)=c^{k}(\underline{\operatorname{rank}}(X))$;

(ii) If $\tau^{k}(X) \neq 0$ for some $k \in \mathbb{Z}$ and $\underline{\text { rank }}(X)$ is contained in $\Delta^{+}(C)$, then $\underline{\operatorname{rank}}\left(\tau^{k}(X)\right)$ is in $\Delta^{+}(C)$. 
Proposition 4.11. For a Frobenius-type triangular matrix algebra $\Lambda$ and $a \Lambda$-module $X$, if either $X \cong \tau^{-k}\left(P_{i}\right)$ or $X \cong \tau^{k}\left(I_{i}\right)$ for some $k \geq 0$ and $1 \leq i \leq n$, the following statements hold:

(i) $X$ is $\tau$-locally free and rigid;

(ii) $\underline{\operatorname{rank}}(X) \in \Delta_{r e}^{+}(C)$;

(iii) If either $Y \cong \tau^{-m}\left(P_{j}\right)$ or $Y \cong \tau^{m}\left(I_{j}\right)$ for some $m \geq 0$ and $1 \leq j \leq n$ with $\underline{\operatorname{rank}}(X)=$ $\underline{\operatorname{rank}}(Y)$, then $X \cong Y$.

Theorem 4.12. For a Frobenius-type triangular matrix algebra $\Lambda$, the following statements hold:

(a) The number of isomorphism classes of indecomposable $\tau$-locally free $\Lambda$-modules is finite if and only if $C$ is of Dynkin type.

(b) If $C$ is of Dynkin type, then the mapping rank $: X \mapsto \underline{\operatorname{rank}}(X)$ induces a bijection between the set of isomorphism classes of indecomposable $\tau$-locally free $\Lambda$-modules and the set of positive roots of the quadratic form $q_{C}(x)$.

Proof. In the case that $C$ is not of Dynkin type, by Lemma 4.2, Lemma 4.6 and Proposition 4.10 , we know there are infinite many isomorphism classes of indecomposable $\tau$-locally free $\Lambda$-modules.

In the case that $C$ is of Dynkin type, firstly, we need to prove that $\underline{\operatorname{rank}}(X) \in \Delta^{+}(C)$ for any indecomposable $\tau$-locally free $\Lambda$-module $X$. We denote $\underline{\operatorname{rank}}(X)=\underline{x}$. By Lemma 4.4 there exists a least $s$ such that $c^{s} \underline{x}>0$ but $c^{s+1} \underline{x} \ngtr 0$. Because $c^{+}=s_{n} \cdots s_{1}$, there also exists a least $i$ such that $0 \leq i \leq n-1, s_{i} \cdots s_{1} c^{t} \underline{x}>0$, but $s_{i+1} \cdots s_{1} c^{t} \underline{x} \ngtr 0$.

We know that $X^{\prime}=F_{i}^{+} \cdots F_{1}^{+} C^{+t} X$ is indecomposable $\tau$-locally free by Lemma 4.9 and that

$$
\underline{\operatorname{rank}}\left(F_{i}^{+} \cdots F_{1}^{+} C^{+t} X\right)=s_{i} \cdots s_{1} c^{t} \underline{x} .
$$

Because $s_{i+1}\left(\underline{\operatorname{rank}}\left(X^{\prime}\right)\right) \ngtr 0$, there is an isomorphism $X^{\prime} \cong E_{i+1}$ by Lemma 4.9. So $s_{i} \cdots s_{1} c^{t} \underline{x}=\alpha_{i+1}$, and according to Lemma 4.3 the vector $\underline{x}=c^{-t} s_{1} \cdots s_{i} \alpha_{i+1}=c^{-t} \beta_{i+1}$ is a positive root of $q_{C}(x)$ and $\underline{\operatorname{rank}}(-)$ is surjective.

If $X$ and $Y$ are indecomposable $\tau$-locally free $\Lambda$-modules such that $\underline{\operatorname{rank}}(X)=\underline{\operatorname{rank}}(Y)$, then we have, as earlier

$$
F_{i}^{+} \cdots F_{1}^{+} C^{+t} X \cong E_{i+1} \cong F_{i}^{+} \cdots F_{1}^{+} C^{+t} Y
$$

so that

$$
X \cong C^{-t} F_{1}^{-} \cdots F_{i}^{-} E_{i+1} \cong Y .
$$

Thus $\underline{\operatorname{rank}}(-)$ is an injective mapping.

Corollary 4.13. For the path algebra $\Lambda=A Q$ of an acyclic quiver $Q$ over a Frobenius algebra $A$, the following statements hold: 
(a) The number of isomorphism classes of indecomposable $\tau$-locally free $\Lambda$-modules is finite if and only if $Q$ is of Dynkin type;

(b) In the case when $Q$ is of Dynkin type, the mapping $\underline{\operatorname{rank}}: X \mapsto \underline{\operatorname{rank}}(X)$ induces a bijection between the set of isomorphism classes of indecomposable $\tau$-locally free $\Lambda$-modules and the set of positive roots of the quadratic form $q_{Q}(x)=\sum_{i \in Q_{0}} x_{i}^{2}-\sum_{\alpha \in Q_{1}} x_{s(\alpha)} x_{t(\alpha)}$, where $x=\left(x_{1}, \ldots, x_{n}\right)^{t} \in \mathbb{Z}^{n}$.

Proof. By Remark 1.2 (iii), $\Lambda=A Q$ is a Frobenius-type triangular matrix algebra via taking all $A_{i}=A$ and $B_{i j}=\oplus_{s=1}^{\#\{\alpha: j \rightarrow i\}} A$. So, $c_{i}=c_{j}=\operatorname{dim}_{k} A$ and then

$$
-c_{i j}=-c_{j i}=\operatorname{dim}_{k} B_{i j} / \operatorname{dim}_{k} A=\#\{\alpha: j \rightarrow i\}
$$

which means that the Cartan matrix $C$ is symmetric and in its corresponding valued quiver $\Gamma(\Lambda)$, the valuation $\left(-c_{i j},-c_{j i}\right)$ is given with the number of arrows from $j$ to $i$ in $Q$. By the definitions of the quadratic forms, we have:

$$
q_{C}(x)=\sum_{i=1}^{n} c_{i} x_{i}^{2}-\sum_{i<j} c_{i}\left|c_{i j}\right| x_{i} x_{j}=\operatorname{dim}_{k} A\left(\sum_{i=1}^{n} x_{i}^{2}-\sum_{i<j}\left|c_{i j}\right| x_{i} x_{j}\right)=q_{Q}(x) \operatorname{dim}_{k} A .
$$

Hence, the positivity definite property of $q_{C}$ and $q_{Q}$ are the same with each other. Thus, the statements (a) and (b) follow respectively from Theorem 4.12 (a) and (b).

Recall in [15] that for a generalized path algebra $\Lambda=k(Q, \mathcal{A})$, there is a corresponding valued quiver $\Upsilon(\Lambda)$, whose the set of vertices $\Upsilon(\Lambda)_{0}=Q_{0}$ and give an arrow $i \leftarrow j$ of $\Upsilon(\Lambda)$, whose valuation is $\left(d_{j i}, d_{i j}\right)$ with $d_{j i}=\left|Q_{i j}\right| \operatorname{dim}_{k} A_{i}$ and $d_{i j}=\left|Q_{i j}\right| d_{i m_{k}} A_{j}$ where $\left|Q_{i j}\right|$ means the number of arrows from $j$ to $i$ in $Q$.

In the case when $Q$ is acyclic, in order to realize $k(Q, \mathcal{A})$ as a triangular matrix algebra, we re-arrange the order of vertices in $Q$ via assuming $i<j$ if there exists a path from $j$ to $i$. Let $B_{i j}=A_{i} Q_{i j} A_{j}$ for $Q_{i j}$ the set of arrows from $j$ to $i$ in $Q$ and then define $A_{i j}$ as in Definition 1.1 and put $A_{i}$ at the $(i, i)$-array. Then we obtain the triangular matrix algebra which is equal to $\Lambda=k(Q, \mathcal{A})$.

Moreover, $-c_{i j}=\operatorname{rank}_{A_{i}}\left(B_{i j}\right)=\left|Q_{i j}\right| \operatorname{dim}_{k} A_{j}=d_{i j}$. Thus, it follows that the valued quiver $\Upsilon(\Lambda)$ is coincident with $\Gamma(\Lambda)$.

Define a quadratic form $q_{k(Q, \mathcal{A})}: \mathbb{Z}^{n} \rightarrow \mathbb{Z}$ of $k(Q, \mathcal{A})$ satisfying for $x=\left(x_{1}, \cdots, x_{n}\right)^{t} \in \mathbb{Z}^{n}$,

$$
q_{k(Q, \mathcal{A})}(x)=\sum_{i \in Q_{0}} d_{i} x_{i}^{2}-\sum_{\alpha \in Q_{1}} d_{s(\alpha)} d_{s(\alpha) t(\alpha)} x_{s(\alpha)} x_{t(\alpha)} .
$$

Comparing this quadratic form with that in (10), it is easy to see that $q_{k(Q, \mathcal{A})}$ is the special case of $q_{C}$ for $\Lambda=k(Q, \mathcal{A})$.

Corollary 4.14. For an acyclic quiver $Q$ and its generalized path algebra $\Lambda=k(Q, \mathcal{A})$ endowed by Frobenius algebras $A_{i}$ at all vertices $i \in Q_{0}$, the following statements hold: 
(a) The number of isomorphism classes of indecomposable $\tau$-locally free $\Lambda$-modules is finite if and only if $\Upsilon(\Lambda)$ is of Dynkin type.

(b) If $\Omega(\Lambda)$ is of Dynkin type, then the mapping rank $: X \mapsto \underline{\text { rank }}(X)$ induces a bijection between the set of isomorphism classes of indecomposable $\tau$-locally free $\Lambda$-modules and the set of positive roots of the $q_{k(Q, \mathcal{A})}(x)=\sum_{i \in Q_{0}} d_{i} x_{i}^{2}-\sum_{\alpha \in Q_{1}} d_{s(\alpha)} d_{t(\alpha)} x_{s(\alpha)} x_{t(\alpha)}$, where $x=$ $\left(x_{1}, \ldots, x_{n}\right)^{t} \in \mathbb{Z}^{n}$ and $d_{i}=\operatorname{dim}\left(A_{i}\right)$.

Proof. They follow directly from $\Upsilon(\Lambda)=\Gamma(\Lambda)$, Fact 4.1 , Theorem 4.12 and that $q_{k(Q, \mathcal{A})}=q_{C}$ for $\Lambda=k(Q, \mathcal{A})$.

\section{Analog of APR-Tilting module For $\Lambda$}

The APR-tilting modules were introduced by Auslander, Platzeck and Reiten in [4] to interpret $B G P$-reflection functors as homomorphism functors of certain tilting modules. Also, it was the beginning of tilting theory.

For a Frobenius-type triangular matrix algebra $\Lambda$, for the case $i=1$, we define $T_{1}:=$ $\Lambda / P_{1} \oplus \tau^{-}\left(P_{1}\right)$ and call $T_{1}$ a generalized APR-tilting module of $\Lambda$. This case follows from the fact that $i=1$ is a "sink vertex" so as to gain the reflection functor.

Proposition 5.1. For a Frobenius-type triangular matrix algebra $\Lambda, T_{1}$ is a tilting $\Lambda$-module.

Proof. Since $P_{1}$ is a rigid and locally free, $\tau^{-}\left(P_{1}\right)$ is rigid and locally free by Proposition 4.8. So $T_{1}$ is locally free. Then by Corollary 2.8 , proj.dim $T_{1} \leq 1$. Thus, $\operatorname{Ext}_{\Lambda}^{1}\left(T_{1}, T_{1}\right) \cong$ $\operatorname{DHom}_{\Lambda}\left(T_{1}, \tau\left(T_{1}\right)\right)=\operatorname{DHom}_{\Lambda}\left(T_{1}, P_{1}\right)=0$. Because $\Lambda$ is connected, $P_{1}$ have no injective summand. Since $\tau^{-}$take non-injective indecomposable module to non-projective indecomposable module, $T_{1}:=\Lambda / P_{1} \oplus \tau^{-}\left(P_{1}\right)$ has the same number of summands as primitive idempotents of $\Lambda$. So $T_{1}$ is a tilting $\Lambda$-module.

Remark 5.2. A similar analog of generalized APR-tilting modules was introduced in [17] for a class of triangular matrix alegbras.

Lemma 5.3. For a Frobenius-type triangular matrix algebra $\Lambda$, there is an algebra isomorphism $\operatorname{End}_{\Lambda}\left(T_{1}\right) \cong S_{1}(\Lambda)$.

Proof. Clearly, when $2 \leq i, j \leq n$

$$
\begin{aligned}
e_{i}^{\prime} \operatorname{End}_{\Lambda}\left(T_{1}\right) e_{j}^{\prime} & \cong \operatorname{Hom}_{\operatorname{End}\left(T_{1}\right)}\left(\operatorname{End}_{\Lambda}\left(T_{1}\right) e_{i}^{\prime}, \operatorname{End}_{\Lambda}\left(T_{1}\right) e_{j}^{\prime}\right) \\
& \cong \operatorname{Hom}_{\operatorname{End}\left(T_{1}\right)}\left(\operatorname{Hom}\left(T_{1}, P_{i}\right), \operatorname{Hom}\left(T_{1}, P_{j}\right)\right) \\
& \cong \operatorname{Hom}_{\Lambda}\left(P_{i}, P_{j}\right) \\
& \cong e_{i} \Lambda e_{j} .
\end{aligned}
$$

When $i=1, j=1, e_{i}^{\prime} \operatorname{End}_{\Lambda}\left(T_{1}\right) e_{j}^{\prime} \cong \operatorname{Hom}_{\Lambda}\left(\tau^{-}\left(P_{1}\right), \tau^{-}\left(P_{1}\right)\right) \cong \operatorname{Hom}_{\Lambda}\left(P_{1}, P_{1}\right) \cong A_{1}$.

When $i=1, j>1, e_{i}^{\prime} \operatorname{End}_{\Lambda}\left(T_{1}\right) e_{j}^{\prime} \cong \operatorname{Hom}_{\Lambda}\left(\tau^{-}\left(P_{1}\right), P_{j}\right)=0$.

When $j=1, i>1, e_{i}^{\prime} \operatorname{End}_{\Lambda}\left(T_{1}\right) e_{j}^{\prime} \cong \operatorname{Hom}_{\Lambda}\left(P_{i}, \tau^{-}\left(P_{1}\right)\right)$. 
Since there is an minimal injective resolution:

$$
0 \rightarrow P_{1} \rightarrow I_{1} \rightarrow \oplus_{j=2}^{n} I_{j} \otimes B_{j 1} \rightarrow 0
$$

then we have

$$
0 \rightarrow \nu^{-}\left(P_{1}\right) \rightarrow P_{1} \rightarrow \oplus_{j=2}^{n} P_{j} \otimes B_{j 1} \rightarrow \tau^{-}\left(P_{1}\right) \rightarrow 0 .
$$

Applying functor $\operatorname{Hom}_{\Lambda}\left(P_{i},-\right)$ for $i>1$.

Since $\operatorname{Hom}_{\Lambda}\left(P_{i}, P_{1}\right)=0, \operatorname{Hom}_{\Lambda}\left(P_{i}, \oplus_{k=2}^{n} P_{k} \otimes B_{k 1}\right) \rightarrow \operatorname{Hom}_{\Lambda}\left(P_{i}, \tau^{-}\left(P_{1}\right)\right)$ is injective.

Since $P_{i}$ is projective $\Lambda$-module, $\operatorname{Hom}_{\Lambda}\left(P_{i}, \oplus_{k=2}^{n} P_{k} \otimes B_{k 1}\right) \rightarrow \operatorname{Hom}_{\Lambda}\left(P_{i}, \tau^{-}\left(P_{1}\right)\right)$ is surjective.

So, $\operatorname{Hom}_{\Lambda}\left(P_{i}, \oplus_{k=2}^{n} P_{k} \otimes B_{k 1}\right) \cong \operatorname{Hom}_{\Lambda}\left(P_{i}, \tau^{-}\left(P_{1}\right)\right)$.

Then, $e_{i}^{\prime} \operatorname{End}_{\Lambda}\left(T_{1}\right) e_{1}^{\prime} \cong \operatorname{Hom}_{\Lambda}\left(P_{i}, \oplus_{k=2}^{n} P_{k} \otimes B_{k 1}\right) \cong \oplus_{k=2}^{n} e_{1} \Lambda e_{k} \otimes B_{k 1} \cong A_{i 1}$.

At last, $\operatorname{End}_{\Lambda}\left(T_{1}\right) \cong S_{1}(\Lambda)$.

Using Proposition 5.1 and Lemma 5.3, we can prove the following theorem.

Theorem 5.4. For a Frobenius-type triangular matrix algebra $\Lambda$, there is a functorial isomorphism

$$
F_{1}^{+}(-) \cong \operatorname{Hom}_{\Lambda}\left(T_{1},-\right): \operatorname{rep}(\Lambda) \rightarrow \operatorname{rep}\left(S_{1}(\Lambda)\right)
$$

Proof. We know that $P_{1} \cong E_{1}$.

Since we have $\nu^{-}\left(D\left(e_{1} \Lambda\right)\right)=\operatorname{Hom}_{\Lambda}\left(D\left(D\left(e_{1} \Lambda\right)\right), \Lambda\right) \cong \operatorname{Hom}_{\Lambda}\left(e_{1} \Lambda, \Lambda\right) \cong \Lambda e_{1}=P_{1}$.

Also

$$
\begin{aligned}
\nu^{-}\left(D\left(B_{1 j} \otimes_{A_{j}} e_{j} \Lambda\right)\right) & =\operatorname{Hom}_{\Lambda}\left(B_{1 j} \otimes_{A_{j}} e_{j} \Lambda, \Lambda\right) \\
& \cong \operatorname{Hom}_{A_{j}}\left(B_{1 j}, \operatorname{Hom}_{\Lambda}\left(e_{j} \Lambda, \Lambda\right)\right) \\
& \cong \operatorname{Hom}_{A_{j}}\left(B_{i j}, \Lambda e_{j}\right) \\
& \cong \Lambda e_{j} \otimes_{A_{j}} \operatorname{Hom}_{A_{j}}\left(B_{1 j}, A_{j}\right) \\
& \cong P_{j} \otimes_{A_{j}} B_{j i} .
\end{aligned}
$$

Since $\Lambda e_{j}$ is a finite generated projective right $A_{j}$-module,the isomorphism in (15) comes from [2]. So applying the quasi-inverse Nakayama functor $\nu^{-}$to (6) we get an exact sequence

$$
0 \rightarrow \nu^{-}\left(E_{1}\right) \rightarrow P_{1} \rightarrow \oplus_{j=2}^{n} P_{j} \otimes_{A_{j}} B_{j i} \rightarrow \tau^{-}\left(P_{1}\right) \rightarrow 0
$$

where $\theta_{1 j}: P_{1} \rightarrow P_{1} \otimes B_{j 1}$ is given by $\lambda e_{i} \mapsto \Sigma_{r \in R_{i j}} \lambda r \otimes r^{*}$. We have isomorphism $H_{o m}\left(P_{j} \otimes\right.$ $\left.B_{j 1}, X\right) \cong \operatorname{Hom}_{\Lambda}\left(\operatorname{Hom}_{A_{j}}\left(B_{i j}, \Lambda e_{j}\right), X\right) \cong B_{1 j} \otimes \operatorname{Hom}_{\Lambda}\left(P_{j}, X\right) \cong B_{1 j} \otimes X_{j}$. The isomorphism $H_{o m}\left(P_{j} \otimes B_{j 1}, X\right) \rightarrow B_{1 j} \otimes X_{j}$ is given by $f \mapsto \Sigma_{r \in R_{i j}} \lambda r \otimes f\left(e_{j} \otimes r^{*}\right)$. And isomorphism 
$\operatorname{Hom}_{\Lambda}\left(P_{1}, X\right) \rightarrow X_{1}$ is given by $g \mapsto g\left(e_{1}\right)$. We get a commutative diagram

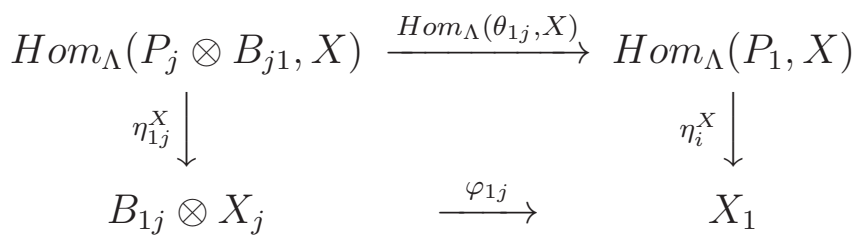

This follows from that for $f \in H_{0} m_{\Lambda}\left(P_{j} \otimes B_{j 1}, X\right)$ and $r \in R_{1 j}$ we have

$$
f\left(r \otimes r^{*}\right)=\varphi_{1 j}\left(r \otimes f\left(e_{j} \otimes r^{*}\right) .\right.
$$

Applying functor $\operatorname{Hom}_{\Lambda}(-, X)$ to (17) for $X \in \Lambda-\bmod$, we obtain a commutative diagram:

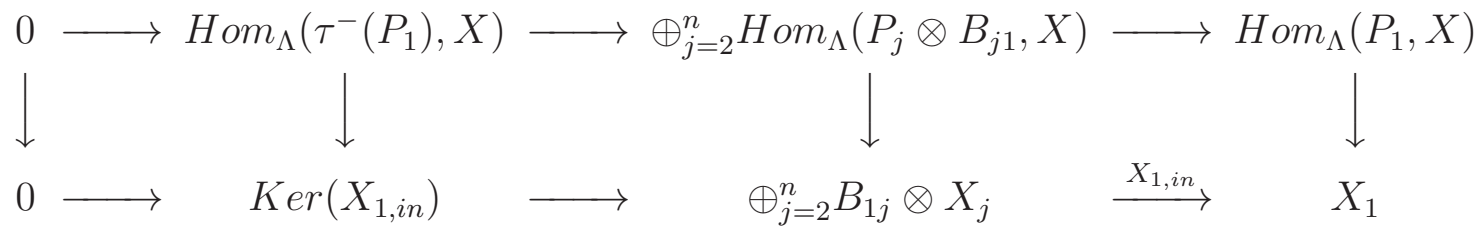

Since the last two terms are isomorphism, we obtain isomorphism $\operatorname{Hom}_{\Lambda}\left(\tau^{-}\left(P_{1}\right), X\right) \cong$ $\operatorname{Ker}\left(M_{1, i n}\right)$. Together with Lemma 5.3, we get the functorial isomorphism $F_{1}^{+}(-) \cong \operatorname{Hom}_{\Lambda}\left(T_{1},-\right)$.

This theorem is the main result in this section, whose corresponding analog in [11] is the [11, Theorem 9.7]. But the method for proving in [11] is incomplete for our case, the Frobenius-type triangular matrix algebra $\Lambda$.

Besides Corollary 4.14, the main results in this paper, including those in this section, are interesting to be restricted two special cases, that is, $\Lambda$ is either a generalized path algebra $\Lambda=k(Q, \mathcal{A})$ endowed by Frobenius algebras $A_{i}$ at each vertex $i \in Q_{0}$ or a path algebra $\Lambda=A Q$ of quiver $Q$ over a Frobenius algebra $A$.

Acknowledgements. We would like to thank Christof Geiss and Jan Schröer for their valuable comments and useful advices. This project is supported by the National Natural Science Foundation of China (No.11271318, No.11571173 and No.J1210038) and the Zhejiang Provincial Natural Science Foundation of China (No.LZ13A010001).

\section{REFERENCES}

[1] Takahide Adachi, Osamu Iyama, and Idun Reiten, $\tau$-tilting theory, Compos. Math. 150 (2014), no. 3, 415-452. MR 3187626

[2] Frank W. Anderson and Kent R. Fuller, Rings and categories of modules, second ed., Graduate Texts in Mathematics, vol. 13, Springer-Verlag, New York, 1992. MR 1245487 (94i:16001)

[3] Ibrahim Assem, Daniel Simson, and Andrzej Skowroński, Elements of the representation theory of associative algebras. Vol. 1, London Mathematical Society Student Texts, vol. 65, Cambridge University Press, Cambridge, 2006, Techniques of representation theory. MR 2197389 (2006j:16020) 
[4] Maurice Auslander, María Inés Platzeck, and Idun Reiten, Coxeter functors without diagrams, Trans. Amer. Math. Soc. 250 (1979), 1-46. MR 530043 (80c:16027)

[5] I. N. Bernšteĭn, I. M. Gel'fand, and V. A. Ponomarev, Coxeter functors, and Gabriel's theorem, Uspehi Mat. Nauk 28 (1973), no. 2(170), 19-33. MR 0393065 (52 \#13876)

[6] Vlastimil Dlab and Claus Michael Ringel, On algebras of finite representation type, J. Algebra 33 (1975), 306-394. MR 0357506 (50 \#9974)

[7] _ Indecomposable representations of graphs and algebras, Mem. Amer. Math. Soc. 6 (1976), no. 173, v+57. MR 0447344 (56 \#5657)

[8] Peter Gabriel, Unzerlegbare Darstellungen. I, Manuscripta Math. 6 (1972), 71-103; correction, ibid. 6 (1972), 309. MR 0332887 (48 \#11212)

[9] _ Auslander-Reiten sequences and representation-finite algebras, Representation theory, I (Proc. Workshop, Carleton Univ., Ottawa, Ont., 1979), Lecture Notes in Math., vol. 831, Springer, Berlin, 1980, pp. 1-71. MR 607140 (82i:16030)

[10] — Auslander-Reiten sequences and representation-finite algebras, Representation theory, I (Proc. Workshop, Carleton Univ., Ottawa, Ont., 1979), Lecture Notes in Math., vol. 831, Springer, Berlin, 1980, pp. 1-71. MR 607140 (82i:16030)

[11] Christof Geiss, Bernard Leclerc, and Jan Schröer, Quivers with relations for symmetrizable cartan matrices I : Foundations, arXiv:1410.1403, 2015, pp. 1-67.

[12] Yasuo Iwanaga, On rings with finite self-injective dimension, Comm. Algebra 7 (1979), no. 4, 393-414. MR 522552 (80h:16024)

[13] V.G. Kac, Infinite-dimensional Lie algebras, Cambridge university press, (1994).

[14] T.Y.Lam, Serre's problem on projective modules, Springer Science \& Business Media, (2010).

[15] Fang Li, Modulation and natural valued quiver of an algebra, Pacific Journal of Mathematics (2012), 256(1): 105-128.

[16] _ and Chang Ye, Gorenstein Projective Modules Over a Class of Generalized Matrix Algebras and their Applications, Algebr. Represent. Theory 18 (2015), no. 3, 693-710. MR 3357944

[17] Liping Li, Stratifications of finite directed categories and generalized APR tilting modules, Comm. Algebra 43 (2015), no. 5, 1723-1741. MR 3316816

[18] Claus Michael Ringel, Representations of K-species and bimodules, J. Algebra 41 (1976), no. 2, 269-302. MR 0422350 (54 \#10340)

[19] —and $\mathrm{Pu}$ Zhang, Representations of quivers over the algebra of dual numbers, arXiv preprint arXiv:1112.1924 (2011).

[20] Meihua Shi and Fang Li, The category of modules over a triangular matrix ring of order $n$ and its homological characterizations, Acta Math. Sinica (Chin. Ser.) 49 (2006), no. 1, 215-224. MR 2249410 (2007f:16013)

FANG Li

Department of Mathematics, Zhejiang University (Yuquan Campus), Hangzhou, Zhejiang 310027, P.R.CHINA

E-mail address: fangli@zju.edu.cn 
Chang Ye

Department of Mathematics, Zhejiang University (Yuquan Campus), HangZhou, Zhejiang 310027, P.R.CHINA

E-mail address: yc2009@zju.edu.cn 